Metin Tolan

\title{
X-Ray Scattering from Soft-Matter Thin Films
}

Materials Science and Basic Research

With 98 Figures

Springer 


\section{Contents}

1. Introduction $\ldots \ldots \ldots \ldots \ldots \ldots \ldots \ldots \ldots \ldots \ldots \ldots \ldots \ldots \ldots \ldots \ldots, 1$

2. Reflectivity of X-Rays from Surfaces.............. 5

2.1 Basic Principles ........................... 5

2.2 Multiple Interfaces............................ 11

2.3 Roughness $\ldots \ldots \ldots \ldots \ldots \ldots \ldots \ldots \ldots \ldots \ldots \ldots \ldots \ldots \ldots \ldots$

2.4 Arbitrary Density Profiles .................... 26

3. Reflectivity Experiments $\ldots \ldots \ldots \ldots \ldots \ldots \ldots \ldots \ldots \ldots \ldots \ldots$

3.1 Experimental Considerations $\ldots \ldots \ldots \ldots \ldots \ldots \ldots \ldots \ldots, 33$

3.1.1 Resolution Functions .................... 36

3.1.2 Data Correction and Parameter Refinement........ 41

3.2 Examples of Soft-Matter Thin Film Reflectivity ......... 43

3.2.1 Polymer Films ....................... 44

3.2.2 Liquid Films on Solid Substrates .............. 58

3.2.3 Liquid Films on Liquid Surfaces $\ldots \ldots \ldots \ldots \ldots \ldots \ldots 65$

3.2.4 Langmuir-Blodgett Films $\ldots \ldots \ldots \ldots \ldots \ldots \ldots \ldots, 68$

4. Advanced Analysis Techniques.................. 75

4.1 The Kinematical Approximation ................. 75

4.1.1 Asymmetric Profiles: A Closer Look ............ 78

4.1 .2 Unique Profiles....................... 80

4.2 The "Phase-Guessing" Inversion Method ............ 81

4.3 Other Data Inversion Techniques ................ 86

5. Statistical Description of Interfaces ................ 91

5.1 Correlation Functions ........................ 91

5.2 Transformation to Reciprocal Space................ 94

5.3 Some Examples ........................... 96

5.3.1 Self-Affine Surfaces ...................... 96

5.3.2 $\quad K$-Correlation Functions $\ldots \ldots \ldots \ldots \ldots \ldots \ldots \ldots, 98$

5.3.3 Capillary Waves and Polymer Surfaces ........... 100

5.4 Vertical Roughness Correlations ................... 109 
6. Off-Specular Scattering $\ldots \ldots \ldots \ldots \ldots \ldots \ldots \ldots \ldots \ldots \ldots \ldots \ldots$

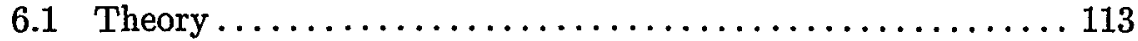

6.1.1 Kinematical Formulation .................. 114

6.1.2 Distorted-Wave Born Approximation ........... 116

6.1.3 Soft-Matter Surfaces ........................ 119

6.2 Experiments .............................. 123

6.2.1 Polymer Films ......................... 124

6.2 .2 Liquid Thin Films ....................... 132

6.2.3 Langmuir-Blodgett Films ................ 135

6.2.4 Roughness Propagation in Soft-Matter Films ....... 139

7. X-Ray Scattering with Coherent Radiation ........... 151

7.1 Coherent versus Incoherent Scattering.............. 152

7.2 General Formalism .......................... 154

7.3 Coherent Scattering from Surfaces .................. 160

7.4 Future Developments ....................... 165

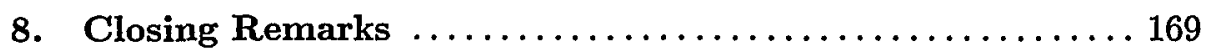

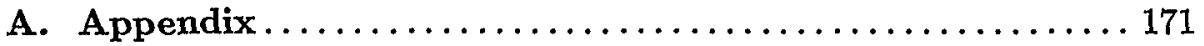

A.1 The Hilbert Phase of Reflection Coefficients............ 171

A.2 The Formalism of the DWBA $\ldots \ldots \ldots \ldots \ldots \ldots \ldots \ldots \ldots \ldots$

A.3 Exact Formulas for Coherent X-Ray Scattering .......... 174

A.4 Diffusive Particle Motion and XPCS ................ 176

References.................................... 179

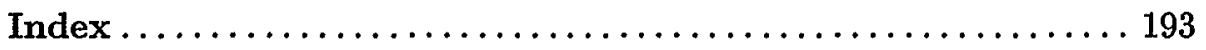

